\title{
A Test of Target Independence of the 'Proton Spin' Effect in Semi-Inclusive Deep Inelastic Scattering
}

\author{
G.M. Shore ${ }^{a * \dagger}$ \\ a Department of Physics, University of Wales Swansea, \\ Singleton Park, Swansea SA2 8PP, U.K.
}

The target-independent suppression mechanism proposed as an explanation of the EMC-SMC 'proton spin' effect is reviewed and compared to current experimental data. According to this proposal, the anomalous suppression observed in the first moment of the polarised proton structure function $g_{1}^{p}$ is not a special property of the proton structure, but reflects an anomalously small value of the first moment of the QCD topological susceptibility. It is shown how this mechanism can be tested in semi-inclusive DIS processes in which a pion or $D$ meson carrying a large target energy fraction $z$ is detected in the target fragmentation region.

\section{Introduction}

The so-called 'proton spin' problem, viz. the anomalous suppression in the first moment $\Gamma_{1}^{p}$ of the polarised structure function $g_{1}^{p}$, continues to be the focus of intense theoretical and experimental activity. In the explanation proposed in collaboration with S. Narison and G. Veneziano[1].2], this suppression is identified as a generic, targetindependent feature of QCD related to the axial anomaly and not a special property of the proton structure.3 The violation of the EllisJaffe sum rule for the flavour singlet form factor $G_{A}^{(0)}(\Delta \Sigma$ in standard notation) contributing to $\Gamma_{1}^{p}$ is understood as one more example of the class of OZI-violating phenomena characteristic of the flavour-singlet pseudoscalar or pseudovector channels. More specifically, it is shown to reflect an anomalous suppression in the first moment of the QCD topological susceptibility $\chi^{\prime}(0)$.

In our view, this explanation is well-motivated and theoretically convincing. However, in view of the difficulties, both theoretical and especially experimental, in obtaining sufficiently accurate numbers for $\Gamma_{1}^{p}$ to give a conclusive confirmation of our proposed mechanism, it is interesting to

\footnotetext{
*SWAT-96/130

${ }^{\dagger}$ Invited talk at QCD 96, Montpellier, July 1996

${ }^{3}$ It is also emphasised in [1, 2] and elsewhere (e.g. [3]), yet consistently ignored by the experimental community, that the singlet form factor $\Delta \Sigma$ does not measure spin.
}

look for other indirect tests. One characteristic, though not unique, feature of our analysis is the prediction of target independence. In this talk, after briefly reviewing our earlier work and the current experimental status of the $\Gamma_{1}^{p}$ sum rule, I shall describe some ideas [4] on how the hypothesis of target-independent flavour-singlet suppression can be tested experimentally in semiinclusive deep inelastic scattering in which a pion or $D$ meson carrying a large target energy fraction $z$ is detected in the target fragmentation region.

\section{The sum rule for $\Gamma_{1}^{p}$}

The sum rule for the first moment of the polarised proton structure function $g_{1}^{p}$ is ${ }^{\text {D: }}$

$$
\begin{aligned}
\Gamma_{1}^{p}\left(Q^{2}\right) \equiv & \int_{0}^{1} d x g_{1}^{p}\left(x ; Q^{2}\right) \\
= & \frac{1}{6}\left[\left(G_{A}^{(3)}(0)+\frac{1}{\sqrt{3}} G_{A}^{(8)}(0)\right) R\left(\alpha_{s}\right)\right. \\
& \left.\quad+\frac{2}{3} G_{A}^{(0)}\left(0 ; Q^{2}\right) R_{0}\left(\alpha_{s}\right)\right]
\end{aligned}
$$

where $G_{A}^{(a)}\left(k^{2}\right)$ are the form factors in the proton matrix elements of the axial current:

$$
\underline{\left\langle P\left|J_{\mu 5 R}^{a}(k)\right| P\right\rangle=G_{A}^{(a)} \bar{u} \gamma_{\mu} \gamma_{5} u+G_{P}^{(a)} k_{\mu} \bar{u} \gamma_{5} u}
$$

${ }^{4}$ We work throughout in the chiral limit. Since all our results depend smoothly on the light quark mass terms in this limit, 2 , the corrections induced by finite quark masses will be small. 
and $R\left(\alpha_{s}\right)$ and $R_{0}\left(\alpha_{s}\right)$ are known perturbative series. The suffix ' $R$ ' indicates a renormalised composite operator and $a$ is an $S U(3)$ flavour index. Since the flavour singlet current $J_{\mu 5 R}^{0}$ is multiplicatively renormalised, its matrix elements, in particular $G_{A}^{(0)}\left(0 ; Q^{2}\right)$, are renormalisation group (RG) scale dependent, scaling with anomalous dimension $\gamma$, a fact which plays a crucial rôle in the correct interpretation of the 'proton spin' effect.

In the QCD-improved parton model, these form factors are related to the polarised quark and gluon distributions by (see, e.g. [3])

$$
\begin{aligned}
& G_{A}^{(3)}(0)=\frac{1}{2}(\Delta u-\Delta d) \\
& G_{A}^{(8)}(0)=\frac{1}{2 \sqrt{3}}(\Delta u+\Delta d-2 \Delta s) \\
& G_{A}^{(0)}\left(0 ; Q^{2}\right) \equiv \Delta \Sigma=\Delta q^{S}-N_{F} \frac{\alpha_{s}}{2 \pi} \Delta g
\end{aligned}
$$

In this latter expression, $\Delta q^{S}=\Delta u+\Delta d+\Delta s$ is assumed to be RG invariant, while the polarised gluon distribution $\Delta g$ has the complicated RG behaviour necessary to ensure multiplicative scaling of $G_{A}^{(0)}\left(0 ; Q^{2}\right) \quad$ (c.f. [1]).

The Ellis-Jaffe sum rule is simply the OZI prediction,

$\left.G_{A}^{(0)}(0)\right|_{\mathrm{OZI}}=2 \sqrt{3} G_{A}^{(8)}(0)=0.58 \pm 0.02$

which leads to

$\left.\Gamma_{1}^{p}\right|_{Q^{2}=10 \mathrm{GeV}^{2}}=0.170 \pm 0.003$

It is obtained in the parton model by neglecting the gluon distribution $\Delta g$ in the singlet form factor and arguing that the polarised strange quark distribution in the proton vanishes, i.e. $\Delta s=0$.

This sum rule is clearly violated by the experimental data. The most recent published results from the SMC collaboration on the proton structure function [5] are

$\left.\Gamma_{1}^{p}\right|_{Q^{2}=10 \mathrm{GeV}^{2}}=0.136 \pm 0.011$ (stat) \pm 0.011 (syst)

from which we extract

$\left.G_{A}^{(0)}(0)\right|_{Q^{2}=10 G e V^{2}}=0.29 \pm 0.15$

However, a recent analysis by Ball, Forte and Ridolfi[6], taking into account the $Q^{2}$ evolution in extracting $\Gamma_{1}^{p}$ from the data (see also Deshpande in these proceedings) and making a different extrapolation to the unmeasured small $x$ region, leads to a lower central value, $\Gamma_{1}^{p}=0.122 \pm$ $0.013(\exp )+0.011 /-0.005($ th $)$, while increasing the error estimate. Interestingly, they also find support in the data for a decomposition of $G_{A}^{(0)}$ into quark and gluon distributions in which the RG invariant $\Delta q^{S}$ is close to the OZI value, in accord with our expectation (see section 3 and [1,2]) that OZI violations probably reside in the non-RG invariant quantities.

Our prediction, incorporating a QCD spectral sum rule estimate of the first moment of the topological susceptibility, is

$\Gamma_{1}^{p}\left(Q^{2}=10 G e V^{2}\right)=0.143 \pm 0.005$

obtained from

$\left.G_{A}^{(0)}(0)\right|_{Q^{2}=10 G e V^{2}}=0.35 \pm 0.05$

Despite the large experimental errors, our prediction is clearly in good agreement with the quoted SMC result, although the BFR[6] reanalysis of the data would move $\Gamma_{1}^{p}$ uncomfortably low. New data is expected shortly from the SMC collaboration and it will be interesting to see how this affects the comparision of theory with experiment. A conservative assessment at present is that the data unquestionably show an OZI violation in a channel where it is to be expected and of roughly the magnitude that can be inferred, as in [2], from the known OZI breaking in the singlet pseudoscalar channel reflected in the $\eta^{\prime}$ mass. Viewed in this light, there is no 'proton spin' problem, simply a challenge to derive a quantitative prediction from first principles of a subtle, anomaly-related QCD phenomenon.

\section{Target Independence and the Compos- ite Operator/Proper Vertex Method for DIS}

The field-theoretic methods we have used to derive (8), (9) are fully described in [1.2] and summarised in [7. For a generic structure function sum rule, we have the expression

$$
\int_{0}^{1} d x x^{n-1} F\left(x ; Q^{2}\right)
$$




$$
=\sum_{i} \sum_{j} C_{i}^{n}\left(Q^{2}\right)\left\langle 0\left|\mathcal{O}_{i}(0) \tilde{\mathcal{O}}_{j}(0)\right| 0\right\rangle \Gamma_{\tilde{\mathcal{O}}_{j} N \bar{N}}
$$

Here, $C_{i}^{n}$ are the Wilson coefficients and $\mathcal{O}_{i}^{n}$ the lowest twist, spin $n$ operators appearing in the OPE of two electromagnetic currents. The novel feature is our decomposition of the resulting nucleon matrix elements $\left\langle N\left|\mathcal{O}_{i}^{n}\right| N\right\rangle$ into the product of zero-momentum composite operator propagators $\left\langle 0\left|\mathcal{O}_{i}(0) \tilde{\mathcal{O}}_{j}(0)\right| 0\right\rangle$ and proper vertices $\Gamma_{\tilde{\mathcal{O}}_{j} N \bar{N}}$ defined as 1PI with respect to an appropriately chosen set of composite operators $\tilde{\mathcal{O}}_{j}$. It is important to recognise that this decomposition is exact and no approximation is involved in using a finite (in practice small) set of operators. A different choice of the set $\tilde{\mathcal{O}}_{j}$ merely changes the definition of the vertices $\Gamma_{\tilde{\mathcal{O}}_{j} N N}$ - the trick is to choose a basis of operators such that the resulting proper vertices have simple RG properties and ideally are closely related to physical couplings.

In the case of the $\Gamma_{1}^{p}$ sum rule, the relevant proton matrix element (after using the axial anomaly and assuming the absence of a massless pseudoscalar Goldstone boson in the singlet channel) is simply the forward matrix element of the gluon topological density $\left\langle P\left|Q_{R}(0)\right| P\right\rangle$. In fact,

$G_{A}^{(0)}\left(0 ; Q^{2}\right) \bar{u} \gamma_{5} u=\frac{1}{2 M} 2 N_{F}\left\langle P\left|Q_{R}(0)\right| P\right\rangle$

(Here, $Q_{R}$ is the renormalised operator related by a non-multiplicative renormalisation to the bare operator $Q_{B}=\frac{\alpha_{s}}{8 \pi} \operatorname{tr} G^{\mu \nu} \tilde{G}_{\mu \nu}$.) The composite operator basis $\tilde{\mathcal{O}}_{j}$ is then chosen to be the set of renormalised flavour singlet pseudoscalar operators $Q_{R}$ and $\Phi_{5 R}$, where (up to a subtle normalisation factor) the corresponding bare operator $\Phi_{5 B}$ is $i \sum \bar{q} \gamma_{5} q$. We then have

$$
\begin{aligned}
& \left\langle P\left|Q_{R}(0)\right| P\right\rangle \\
& =\left\langle 0\left|Q_{R} Q_{R}\right| 0\right\rangle \Gamma_{Q_{R} P \bar{P}}+\left\langle 0\left|Q_{R} \Phi_{5 R}\right| 0\right\rangle \Gamma_{\Phi_{5 R} P \bar{P}}
\end{aligned}
$$

where the composite operator propagators are at zero momentum and the proper vertices are $1 \mathrm{PI}$ with respect to $Q_{R}$ and $\Phi_{5 R}$ only.

The composite operator propagator in the first term is the zero-momentum limit of the QCD topological susceptibility $\chi\left(k^{2}\right)$, viz.

$$
\chi\left(k^{2}\right)=\int d x e^{i k \cdot x} i\left\langle 0\left|T^{*} Q_{R}(x) Q_{R}(0)\right| 0\right\rangle
$$

The anomalous chiral Ward identities show that $\chi(0)$ vanishes for $\mathrm{QCD}$ with massless quarks, in contrast to pure Yang-Mills theory where $\chi(0)$ is non-zero. Furthermore, it can be shown[1] that the propagator $\left\langle 0\left|Q_{R} \Phi_{5 R}\right| 0\right\rangle$ at zero momentum is simply the square root of the first moment of the topological susceptibility. We therefore find:

$$
\left\langle P\left|Q_{R}(0)\right| P\right\rangle=\sqrt{\chi^{\prime}(0)} \Gamma_{\Phi_{5 R} P \bar{P}}
$$

The quantity $\sqrt{\chi^{\prime}(0)}$ is not $R G$ invariant and scales with the anomalous dimension $\gamma$. On the other hand, the proper vertex has been chosen specifically so as to be RG invariant. The renormalisation group properties of this decomposition are crucial to our resolution of the 'proton spin problem'.

Our proposal (which is fully motivated in [1, 2, 7) is that we should expect the source of OZI violations to lie in the RG non-invariant, and therefore anomaly-sensitive, terms, i.e. in $\chi^{\prime}(0)$. Our quantitative prediction (9) then follows by using the OZI approximation for the vertex $\Gamma_{\Phi_{5 R} P \bar{P}}$ and a QCD spectral sum rule estimate of the first moment of the topological susceptibility. We find 2

$\left.\sqrt{\chi^{\prime}(0)}\right|_{Q^{2}=10 \mathrm{GeV}^{2}}=23.2 \pm 2.4 \mathrm{MeV}$,

a suppression of approx. 0.6 relative to the OZI value $f_{\pi} / \sqrt{6}$.

Since, according to this proposal, the anomalous suppression in $\Gamma_{1}^{p}$ is assigned to the composite operator propagator rather than the proper vertex, the suppression is a target independent property of QCD related to the axial anomaly, not a special property of the proton structure. This immediately raises the question whether it is possible to test our proposed mechanism by (12) effectively performing DIS experiments on other hadronic targets.

\section{Other Targets}

Leaving until section 5 the question of how this may be experimentally realised, we now consider the implications of this target-independent suppression mechanism for structure functions of other hadrons besides the proton and neutron. 
The basic assumption is that for any hadron, the singlet form factor in (1) can be substituted by its OZI value times a universal (targetindependent), RG invariant, suppression factor $s\left(Q^{2}\right)$, i.e.

$\Gamma_{1}=\frac{1}{6} R\left(\alpha_{s}\right)\left[G_{A}^{(3)}(0)+\frac{1}{\sqrt{3}} G_{A}^{(8)}(0)(1+4 s)\right]$

where $s=\frac{R_{0}}{R} \frac{G_{A}^{(0)}}{2 \sqrt{3} G_{A}^{(8)}}$. Since $s$ is target independent, we can use the value measured for the proton to deduce $\Gamma_{1}$ for any other hadron target simply from the flavour non-singlet form factors, which obey relations from flavour $S U(3)$ symmetry. From our calculations, eq.(9), we find $s \sim 0.66$ at $Q^{2}=10 \mathrm{GeV}^{2}$, while the central value of the SMC result (7) gives $s \sim 0.55$.

The form factors for a hadron $\mathcal{B}$ are given by the matrix elements of the flavour octet axial current. The $S U(3)$ properties are summarised by

$$
\begin{aligned}
& \left\langle\mathcal{B}\left|J_{I I_{3} Y}^{(\rho)}\right| \mathcal{B}\right\rangle=\left\langle\rho^{\mathcal{B}}\left|J^{(\rho)}\right| \rho^{\mathcal{B}}\right\rangle\left\langle I^{\mathcal{B}} I_{3}^{\mathcal{B}} ; I^{\overline{\mathcal{B}}} I_{3}^{\overline{\mathcal{B}}} \mid I I_{3}\right\rangle
\end{aligned}
$$

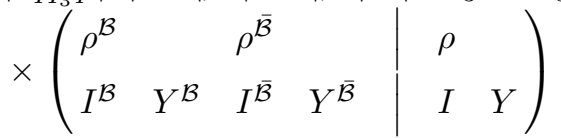

Here, $\rho$ indicates the $S U(3)$ representation while $I, I_{3}$ and $Y$ are the isospin and hypercharge quantum numbers. The term $\left\langle\rho^{\mathcal{B}}\left|J^{(\rho)}\right| \rho^{\mathcal{B}}\right\rangle$ is a reduced matrix element, while the other factors are $S U(2)$ and $S U(3)$ Clebsch-Gordon coefficients.

If we now take the hadron $\mathcal{B}$ to be in the $\mathbf{1 0}$ representation, then since

$10 \times \overline{10}=1+8+27+64$

the matrix element of the (octet) current contains just one reduced matrix element. In contrast to the case of $\mathcal{B}$ in the octet representation as for the proton or neutron, there is no $F / D$ ratio involved (recall $\mathbf{8} \times \mathbf{8}=\mathbf{1}+\mathbf{8}+\mathbf{8}+\mathbf{1 0}+\mathbf{2 7}$ gives two reduced matrix elements). This means that the ratio of $\Gamma_{1}$ for decuplet states can be predicted as a simple group-theoretic number, up to the dynamical suppression factor $s$.

For example, for the $\Delta^{++}$, the matrix element of the current $J_{\mu 5 R}^{3}$ is

$\left\langle\Delta^{++}\left|J_{\mu 5 R}^{3}\right| \Delta^{++}\right\rangle=\sqrt{\frac{3}{10}}\left\langle\mathbf{1 0}\left|J^{\mathbf{8}}\right| \mathbf{1 0}\right\rangle$ evaluating the $\mathrm{C}-\mathrm{G}$ coefficient. A similar result holds for the current $J_{\mu 5 R}^{8}$, with the C-G coefficient being $\sqrt{\frac{1}{10}}$. Combining this with the equivalent results for the $\Delta^{-}$, we find the ratio of the first moment of the polarised structure functions $g_{1}$ for the $\Delta^{++}$and $\Delta^{-}$is

$\frac{\Gamma_{1}^{\Delta^{++}}}{\Gamma_{1}^{\Delta^{-}}}=\frac{\sqrt{\frac{3}{10}}+\sqrt{\frac{1}{10}} \sqrt{\frac{1}{3}}(1+4 s)}{\sqrt{\frac{3}{10}}-\sqrt{\frac{1}{10}} \sqrt{\frac{1}{3}}(1+4 s)}=\frac{2 s+2}{2 s-1}$

The OZI prediction, $s=1$, would therefore be $\Gamma_{1}^{\Delta^{++}} / \Gamma_{1}^{\Delta^{-}}=4$. This can be simply obtained in the valence quark model with the assumption $\Delta u\left(\Delta^{++}\right)=\Delta d\left(\Delta^{-}\right)$. However, substituting a suppression factor of $s \sim 0.66$ gives a much larger ratio $\Gamma_{1}^{\Delta^{++}} / \Gamma_{1}^{\Delta^{-}} \sim 10$, while a value of $s$ nearer 0.5 would give an even bigger value.

We would therefore expect to find a quite spectacular deviation from the quark model expectation for this ratio of structure function moments. We can also show that the same result is obtained for the ratio $\Gamma_{1}^{\Sigma_{c}^{++}} / \Gamma_{1}^{\Sigma_{c}^{0}}$ for the charmed baryons $\Sigma_{c}^{++}=u u c$ and $\Sigma_{c}^{0}=d d c$. Of course, these examples have been specially selected (because of the $2 s-1$ factor) to show a particularly striking difference from the simple quark model predictions. However, as we now see, they are also the examples which can be dynamically isolated in semi-inclusive DIS.

\section{Semi-Inclusive DIS}

The semi-inclusive DIS reaction $\mu N \rightarrow \mu h X$, where $h$ is the detected hadron (a pion or $D$ meson), has distinct contributions from the current and target fragmentation regions, which we assume can be clearly kinematically distinguished. We require $h$ to be in the target fragmentation region and furthermore that the hadron energy fraction $z$ should be large. In this limit, the reaction is well described by the diagram below, in which a meson $h$ carrying a large fraction of the target nucleon energy is emitted with the exchange of a Reggeon $\mathcal{B}$ with well-defined $S U(3)$ quantum numbers. A large rapidity gap is required between $h$ and the inclusive hadrons $X$. With a polarised beam and target, this kinemat- 
ics [- 1 allows us to measure the structure function $g_{1}^{\mathcal{B}}$ of the exchanged Regge trajectory $\mathcal{B}$.

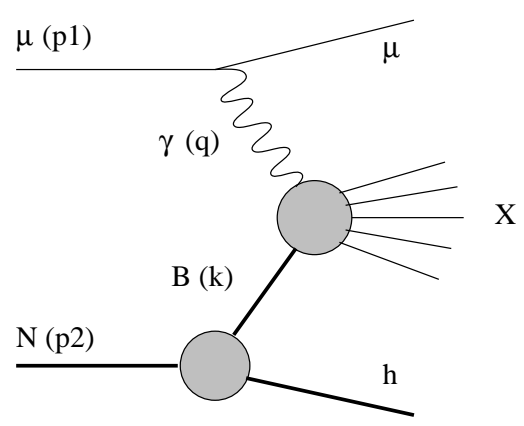

Since the results of section 4 depend solely on the $S U(3)$ properties of the baryon $\mathcal{B}$, they will still hold here despite the fact that $\mathcal{B}$ is interpreted as a Reggeon. If the target is a nucleon $N$ and the detected hadron is an octet meson $(\pi)$, $S U(3)$ symmetry shows that $\mathcal{B}$ belongs to a representation on the rhs of

$8 \times 8=1+8+8+10+\overline{10}+27$

Since the $\mathbf{2 7}$ requires a 5 -quark state, it is a good dynamical approximation that the $\mathbf{1 0}$ dominates the 27. However, there is no such argument for $\mathbf{8}$ dominance over the $\mathbf{1 0}$. To isolate a unique representation for $\mathcal{B}$, we must therefore choose a combination of $N$ and $h$ giving $I_{3}, Y$ quantum numbers for $\mathcal{B}$ which appear in the $\mathbf{1 0}$ but not in the 8 . This is satisfied by the $\Delta^{++}$and $\Delta^{-}$, as in section 4 . The required ratio of first moments $\Gamma_{1}^{\Delta^{++}} / \Gamma_{1}^{\Delta^{-}}=\frac{2 s+2}{2 s-1}$, where now $\Delta^{++}$and $\Delta^{-}$are Reggeons, is therefore obtained by comparing the reactions $\mu p \rightarrow \mu \pi^{-} X$ and $\mu n \rightarrow \mu \pi^{+} X$.

These symmetry considerations are easily pictured [4] by drawing quark diagrams for the $N h \mathcal{B}$ vertex. We also find that the same numerical ratio for the moments arises (as in section 4) in processes in which a $D$ meson is detected, comparing the reactions $\mu p \rightarrow \mu D^{-} X$ and $\mu n \rightarrow \mu D^{0} X$.

The dynamics of these semi-inclusive processes is described in detail in [- The first moment of the polarised structure function for the Reggeon $\mathcal{B}$ is found from the polarisation asymmetry of the differential cross section in the target fragmenta- tion region, i.e.

$$
\begin{aligned}
& \int_{0}^{1-z} d x x \frac{d \Delta \sigma^{\text {target }}}{d x d y d z} \\
& =\frac{Y_{P}}{2} \frac{4 \pi \alpha^{2}}{s} \int d t \Delta f(z, t) \int_{0}^{1} d x_{\mathcal{B}} g_{1}^{\mathcal{B}}\left(x_{\mathcal{B}}, t ; Q^{2}\right)
\end{aligned}
$$

Here, $x=\frac{Q^{2}}{2 p_{2} \cdot q}, x_{\mathcal{B}}=\frac{Q^{2}}{2 k \cdot q}, 1-z=\frac{x}{x_{\mathcal{B}}}, y=$ $\frac{p_{2} \cdot q}{p_{2} \cdot p_{1}}, t=k^{2}$ and $Y_{P}=\frac{1}{y}(2-y)$. If we now take the ratio of the two reactions described above, the factorised Reggeon emission factor $\Delta f(z, t)$ cancels out, leaving the ratio of structure function moments $\Gamma_{1}$ predicted in section 4 to be given simply by the ratio of the cross section moments.

All this can be precisely formulated [4] in the language of fracture functions, which have recently been applied both to unpolarised and polarised semi-inclusive DIS (see [4] for citations). In particular, the 'Reggeon structure function' can be given a precise meaning in terms of a sum over partons $i$ of the (polarised) fracture functions $\Delta M_{i}^{h N}\left(x, z ; Q^{2}\right)$ in the $z \sim 1$ region.

A detailed description of the dynamics of these semi-inclusive processes and some further predictions will be given elsewhere沺. However, this brief sketch should be sufficient to demonstrate that there are exciting possibilities of discovering relations between structure function moments which differ dramatically from simple quark-parton expectations and which could test the target-independent singlet suppression mechanism responsible for the 'proton spin' effect.

\section{REFERENCES}

1. G.M. Shore and G. Veneziano, Phys. Lett. B244 (1990) 75, Nucl. Phys. B381 (1992) 23

2. S. Narison, G.M. Shore and G. Veneziano, Nucl. Phys. B433 (1995) 209

3. G. Altarelli and G. Ridolfi, Nucl. Phys. B (Proc. Suppl.) 39B,C (1995) 106

4. G.M. Shore and G. Veneziano, in preparation.

5. SMC: D. Adams et al., Phys. Lett. B329 (1994) 399.

6. R.D. Ball, S. Forte and G. Ridolfi, Phys. Lett. B378 (1996) 255

7. G.M. Shore, Nucl. Phys. B (Proc. Suppl.) 39B,C (1995) 101 